Article

\title{
Predictors of Vitamin D-Containing Supplement Use in the Australian Population and Associations between Dose and Serum 25-Hydroxyvitamin D Concentrations
}

\author{
Lucinda J. Black ${ }^{1, *}$, Peter Jacoby ${ }^{2}$, Caryl A. Nowson ${ }^{3}$, Robin M. Daly ${ }^{3}$ and Robyn M. Lucas ${ }^{4}$ \\ 1 School of Public Health, Curtin University, Bentley 6102, Australia \\ 2 Telethon Kids Institute, The University of Western Australia, Subiaco 6008, Australia; \\ peter.jacoby@telethonkids.org.au \\ 3 Institute for Physical Activity and Nutrition (IPAN), School of Exercise and Nutrition Sciences, \\ Deakin University, Geelong 3125, Australia; caryl.nowson@deakin.edu.au (C.A.N.); \\ robin.daly@deakin.edu.au (R.M.D.) \\ 4 National Centre for Epidemiology and Population Health, Research School of Population Health, \\ The Australian National University, Canberra 0200, Australia; robyn.lucas@anu.edu.au \\ * Correspondence: lucinda.black@curtin.edu.au; Tel.: +61-8-9266-2523
}

Received: 10 March 2016; Accepted: 30 May 2016; Published: 8 June 2016

\begin{abstract}
Despite concerns about vitamin D deficiency in the Australian population, little is known about the prevalence and predictors of vitamin D-containing supplement use. We described the use of vitamin D-containing supplements, and investigated associations between supplemental vitamin D intake and serum 25-hydroxyvitamin D (25(OH)D) concentrations, using a single 24-h dietary recall from the 2011-2013 Australian Health Survey ( $n=12,153$; ages $\geqslant 2$ years). Multiple regression models were used to investigate predictors of vitamin D-containing supplement use in adults, and associations between dose and serum $25(\mathrm{OH}) \mathrm{D}$ concentrations/vitamin D sufficiency ( $\geqslant 50 \mathrm{nmol} / \mathrm{L}$ ), adjusting for potential confounders. The prevalence of vitamin D-containing supplement use was $10 \%, 6 \%$ and $19 \%$ in children, adolescents and adults, respectively. Predictors of vitamin D-containing supplement use in adults included being female, advancing age, higher educational attainment, higher socio-economic status, not smoking, and greater physical activity. After adjusting for potential confounders, a $40 \mathrm{IU}(1 \mu \mathrm{g})$ increase in vitamin D intake from supplements was associated with an increase of $0.41 \mathrm{nmol} / \mathrm{L}$ in serum $25(\mathrm{OH}) \mathrm{D}$ concentrations $(95 \% \mathrm{CI} 0.35,0.47 ; p<0.001)$. However, the prevalence of vitamin D-containing supplement use was generally low in the Australian population, particularly for single vitamin D supplements, with most supplement users obtaining only low levels of vitamin D from other supplement types.
\end{abstract}

Keywords: vitamin D; supplements; 25-hydroxyvitamin D

\section{Introduction}

Vitamin D deficiency has traditionally not been considered an important public health issue in Australia due to the abundance of sunlight. However, Australia has the highest skin cancer incidence in the world [1] and public health policies have, therefore, emphasised sun protection practices that limit excessive exposure of the skin to ultraviolet radiation. In a similar timeframe, societal changes, particularly the advent of the personal computer, has meant that many Australians now lead predominantly indoor lifestyles. Findings from the 2011 to 2013 Australian Health Survey showed that $23 \%$ of the adult population were vitamin D deficient, defined as serum 25 -hydroxyvitamin $\mathrm{D}$ $(25(\mathrm{OH}) \mathrm{D})$ concentrations $<50 \mathrm{nmol} / \mathrm{L}[2]$. The prevalence of vitamin D deficiency varied by ethnicity, 
and was highest in those born in North Africa, the Middle East and Asia. There is also evidence to suggest a re-emergence of rickets in some Australian children, particularly in recent migrants with dark skin [3].

Although the major source of vitamin D in humans is cutaneous synthesis in the presence of sunlight, when sun exposure is limited (e.g., due to sun protection, working indoors, cultural clothing habits, season and weather conditions), dietary sources of vitamin $\mathrm{D}$ are required to maintain adequate vitamin D status. Given that natural food sources of vitamin D are limited to meat, fish, eggs, dairy and mushrooms, with vitamin D present mostly in small amounts, supplementation is an alternative for increasing vitamin D intakes and status. Vitamin D supplementation has been shown to substantially increase vitamin D intakes and serum $25(\mathrm{OH}) \mathrm{D}$ concentrations in other populations [4-9] and the prevalence of supplement use is increasing globally [10,11]. However, very little is known about the prevalence and predictors of vitamin D-containing supplement use in the Australian population.

The 2011-2013 Australian Health Survey collected comprehensive information on dietary supplement use in a representative sample of the population aged $\geqslant 2$ years, along with serum $25(\mathrm{OH}) \mathrm{D}$ concentrations in those aged $\geqslant 12$ years measured using internationally-standardised methodology [12]. The aims of this study were to describe the prevalence of vitamin D-containing supplement use in the Australian population, identify independent predictors of vitamin D-containing supplement use in adults, and investigate associations between supplemental vitamin D intake and serum 25(OH)D concentrations.

\section{Materials and Methods}

\subsection{Study Population}

The 2011-2013 Australian Health Survey was the largest, most comprehensive health survey ever conducted in Australia, and aimed to provide a better understanding of the health of people living in Australia [12]. It combined the National Health Survey, the National Nutrition and Physical Activity Survey (NNPAS) and the National Health Measures Survey. The survey excluded residents of non-private dwellings and visitors who were not usual residents of the selected dwelling. Each household contributed either one adult, or one adult plus one child/adolescent.

Core data items were collected for all people in the Australian Health Survey and individuals were then selected to participate either in the National Health Survey $(n=20,426)$, which included detailed health measures and use of medications, or in the NNPAS $(n=12,153)$. The NNPAS included a dietary recall of all food, beverages and supplements for participants aged $\geqslant 2$ years. Detailed methodology of the NNPAS is available elsewhere [12]. In brief, trained interviewers collected information by face-to-face interview from usual residents of private dwellings in urban and rural areas of Australia. A second interview was conducted by telephone for a subset of participants. To account for possible seasonal effects, the NNPAS was conducted over a 12-month period from May 2011 to June 2012.

Those aged $\geqslant 5$ years who participated in the National Health Survey or the NNPAS were invited to participate in the National Health Measures Survey for measurement of nutrient and chronic disease biomarkers (urine test for those aged $5-12$ years; blood and urine tests for those aged $\geqslant 12$ years). Qualified phlebotomists collected fasting and non-fasting blood samples from participants aged $\geqslant 12$ years $(n=10,401)$ for measurement of biomarkers, including serum $25(\mathrm{OH}) \mathrm{D}$ concentrations [12]. No participants were excluded from the National Health Measures Survey due to their health status or life stage.

The interview components of the NHS and NNPAS were conducted under the Census and Statistics Act (CSA) 1905. Ethics approval was sought and gained for the NHMS from the Australian Government Department of Health and Ageing's Departmental Ethics Committee (Application Number 2/2011). For the NHMS, informed consent was sought from adults and from parents/legal guardians of children through completion of a consent form. 


\subsection{Use of Vitamin D-Containing Supplements}

For the purposes of this study, we used data from the first 24-h dietary recall of the NNPAS. Participants were asked: "Have you taken any dietary supplements in the last $24 \mathrm{~h}$ ?" and were encouraged to have in front of them any supplements taken [12]. The type, brand and AUST L number [13] of the supplements were recorded, along with the form (e.g., tablet, capsule) and dose. We determined vitamin D composition data primarily from the Australian Register of Therapeutic Goods, a database of therapeutic goods which can be lawfully supplied in Australia [13]. In some cases, the supplements were not listed in the Australian Register of Therapeutic Goods, either because the product has since been relicensed due to name or formulation change, or the product has been taken off the market. In these cases, vitamin D composition data were obtained from manufacturers by website, telephone call, or email communication.

Supplements that contained any vitamin D (either added, or inherent in the case of fish liver oil) were considered a vitamin D-containing supplement. A participant who reported using any supplement that contained vitamin D was considered a "vitamin D-containing supplement user". Vitamin D-containing supplements were classified as single vitamin D supplements, calcium preparations (with added vitamin D), multivitamin/mineral (with added vitamin D), fish oil preparations (with added vitamin D), and fish liver oils (including inherent and added vitamin D).

\subsection{Analysis of Serum 25-Hydroxyvitamin D Concentrations}

The Australian Health Survey is one of eight participating national surveys in the Vitamin D Standardization Program (VDSP), which was established by the National Institute of Health Office of Dietary Supplements in collaboration with the Centers for Disease Control and Prevention and the National Institute for Standards and Technology (NIST). The VDSP aims to internationally standardise the analysis of serum 25(OH)D concentrations [14]. Serum 25(OH)D concentrations for participants aged $\geqslant 12$ years were measured at the Douglass Hanly Moir (DHM) laboratory by a liquid chromatography-tandem mass spectrometry (LC-MS/MS) method. This laboratory is certified to the standard reference method developed by NIST and Ghent University [14].

\subsection{Participant Characteristics}

We categorised age as follows: $2-4,5-8,9-11,12-13,14-15,16-17,18-30,31-50,51-70$ and $\geqslant 71$ years. We defined those aged $2-11$ years, $12-17$ years and $\geqslant 18$ years as children, adolescents and adults, respectively. For adult participants, we described the following characteristics: sex, age group, region of birth, State/Territory, education, socioeconomic status, body mass index (BMI) category, physical activity, smoking, alcohol intake, health condition and self-assessed health.

Region of birth was defined as Australia and New Zealand, Europe, Americas, Asia, Africa and the Middle East. Season was defined as: Summer (December-February), Autumn (March-May), Winter (June-August) and Spring (September-November). State/Territory was defined as New South Wales, Victoria, Queensland, South Australia, Western Australia, Tasmania, Northern Territory and Australian Capital Territory. Educational attainment was described as none after secondary school, certificate, Bachelor/Diploma and postgraduate. Socioeconomic status was described by the Socio-Economic Indexes for Areas (SEIFA) 2011 Index of Relative Socio-Economic Disadvantage (IRSD). This is a general socio-economic index that summarises a range of information about the economic and social conditions of people and households within an area, with scores ranging from low (relatively greater disadvantage in general) to high (relative lack of disadvantage in general) [15]. The SEIFA IRSD was categorised into quintiles.

BMI was calculated from measured weight $(\mathrm{kg})$ and height $(\mathrm{m})$ (weight $/$ height $^{2}$ ), and adults were categorised as underweight (BMI < 18.5), normal weight $(18.5 \leqslant \mathrm{BMI}<25.0)$, overweight $(25.0 \leqslant$ BMI $<30.0)$ or obese (BMI $\geqslant 30)$ [12]. Physical activity was defined as low, moderate, or high based on the level of physical activity for fitness, recreation, sport, or walking for transport in the past 
week [12]. The data items that contributed to this variable included total minutes undertaking moderate exercise/physical activity in the last week; total minutes undertaking vigorous exercise/physical activity in the last week; total minutes walked for fitness, recreation or sport in the last week; and total minutes spent walking for transport in the last week. Each category of physical activity had an intensity factor score (e.g., walking for fitness $=3.5$, walking for transport $=3.5$, moderate exercise $/$ physical activity $=5$ and vigorous exercise/physical activity $=7.5$ ) [12]. The duration of physical activity was multiplied by the intensity factor score. Different levels of exercise/physical activity were defined as: low (no exercise to <800); moderate (800 to 1600, or more than 1600 but with less than $1 \mathrm{~h}$ vigorous physical activity); high (>1600 and with $1 \mathrm{~h}$ or more of vigorous physical activity).

Smoking was defined as "current smoker", "ex-smoker" and "never smoked". Alcohol intake (g/day) was measured by the 24-h dietary recall. Health condition was defined as "yes" or "no" based on whether the participant had ever been told by a medical practitioner that they had one or more of the following conditions (either current or past): diabetes, kidney disease, high cholesterol, high sugar levels, hypertensive disease, ischaemic heart disease, heart failure or other heart disease, cerebrovascular disease, oedema or angina. Self-assessed health was measured as excellent, very good, good, fair or poor.

\subsection{Statistical Analysis}

The prevalence of vitamin D-containing supplement use in the total population was reported by sex, age group and type of supplement used. We determined the proportion of children, adolescents and adults exceeding the Tolerable Upper Intake Level (UL) of vitamin D from supplements on the recording day. The UL is the maximum level of chronic daily intake of a nutrient that is unlikely to pose a risk of adverse health effects to humans [16]. The UL was defined as $2500 \mathrm{IU}(62.5 \mu \mathrm{g})$ for children aged 1-3 years; $3000 \mathrm{IU}(75 \mu \mathrm{g})$ for children aged 4-8 years; and $4000 \mathrm{IU}$ (100 $\mu \mathrm{g})$ for those aged $\geqslant 9$ years, as per the 2011 Institute of Medicine guidelines [17].

We described characteristics of adult vitamin D-containing supplement users and non-users $(n=9435)$, using number and proportion for categorical variables, and median (interquartile range, IQR) for alcohol intake (g/day). Characteristics of users and non-users of vitamin D-containing supplements were compared using Pearson's chi-square tests for categorical variables, and the Mann-Whitney U test for alcohol intake.

We used univariate logistic regression to investigate the relationship between vitamin D-containing supplement use in children/adolescents and adults in the same household ( $n=2634$ households; $n=5268$ individuals). We used multiple logistic regression to investigate the potential predictors of vitamin D-containing supplement use in adults, namely sex, age group, region of birth, State/Territory, season, education, SEIFA, BMI category, physical activity, smoking, alcohol intake, health condition and self-assessed health $(n=7751)$.

We reported the median and IQR for serum $25(\mathrm{OH}) \mathrm{D}$ concentrations in adults (stratified by sex and age group) for those taking no vitamin D-containing supplements, and for those taking <400 IU $(<10 \mu \mathrm{g}), 400-999$ IU $(10-24.9 \mu \mathrm{g})$, and $\geqslant 1000 \mathrm{IU}(\geqslant 25 \mu \mathrm{g})$ of supplemental vitamin D $(n=9435)$. The $400 \mathrm{IU}(10 \mu \mathrm{g})$ cut-off was chosen to reflect the Estimated Average Requirement of vitamin D for for all ages above one year, assuming minimal sunlight exposure [17], while the $1000 \mathrm{IU}$ (25 $\mu \mathrm{g})$ cut-off was chosen to reflect the usual daily dose in single vitamin D supplements.

Using Pearson's chi-square tests, we compared the major characteristics (sex, age group, region of birth, education and SEIFA) of participants with measured serum 25(OH)D concentrations (i.e., those who agreed to have their blood taken for the measurement of biomarkers, $n=3736)$ versus those without measured serum $25(\mathrm{OH}) \mathrm{D}$ concentrations (i.e., those who did not agree to have their blood taken for the measurement of biomarkers, $n=5699)$.

We examined associations between vitamin $\mathrm{D}$ intake from supplements (as a continuous variable) and serum 25(OH)D concentrations in adults using multiple linear regression models. Models were run unadjusted $(n=3736)$ and adjusted for sex, age group, region of birth, State/Territory, education, SEIFA, BMI category, physical activity, smoking, alcohol intake, health condition and self-assessed health $(n=3471)$. Further, we examined the association between vitamin $\mathrm{D}$ intake from supplements 
and vitamin D sufficiency (defined as $\geqslant 50 \mathrm{nmol} / \mathrm{L}$ as per the 2011 Institute of Medicine guidelines [17]) in adults using multiple logistic regression, adjusting for the variables above $(n=3471)$. In order to assess whether the associations varied by sex or by season of blood collection, we also stratified these analyses by sex and season.

Analyses were performed using IBM SPSS Statistics Release Version 19.9.9.1 (IBM SPSS Inc., 2010, Chicago, IL, USA) and statistical significance was defined as two-tailed $p<0.05$.

\section{Results}

\subsection{Prevalence of Vitamin D-Containing Supplement Use}

Overall, 17\% $(n=2039)$ of participants reported using a vitamin D-containing supplement on the recording day: $10 \%(n=176)$ of children, $6 \%(n=63)$ of adolescents and $19 \%(n=1800)$ of adults (Table 1$)$. In adults, there was a clear sex difference in the prevalence of vitamin D-containing supplement use (Figure 1), with $24 \%$ of females and $13 \%$ of males using vitamin D-containing supplements. The characteristics of adult vitamin D supplement users and non-users are shown in Table 2.

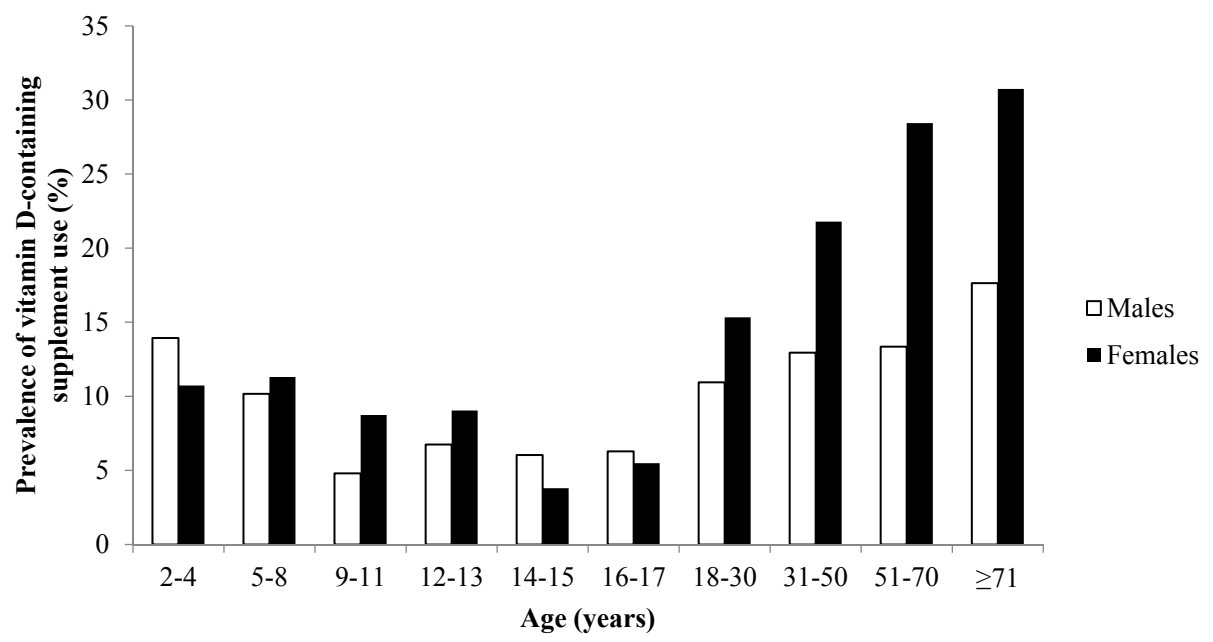

Figure 1. Prevalence of vitamin D-containing supplement use by sex and age group in the Australian population $(n=12,153)$.

Table 1. Prevalence of vitamin D-containing supplement use in the Australian population $(n=12,153)$ by sex, age group and supplement type.

\begin{tabular}{|c|c|c|c|c|c|c|c|c|}
\hline & 2-11 Years & 12-17 Years & 18-30 Years & 31-50 Years & 51-70 Years & $\geqslant 71$ Years & $\geqslant 2$ Years & $\geqslant 18$ Years \\
\hline & $n(\%)$ & $n(\%)$ & $n(\%)$ & $n(\%)$ & $n(\%)$ & $n(\%)$ & $n(\%)$ & $n(\%)$ \\
\hline \multicolumn{9}{|c|}{ Total } \\
\hline All types & $176(10.3)$ & $63(6.3)$ & $224(13.3)$ & $629(17.6)$ & $624(21.5)$ & $323(25.3)$ & 2039 (16.8) & 1800 (19.1) \\
\hline Single vitamin D & $1(0.1)$ & $5(0.5)$ & $26(1.5)$ & $104(2.9)$ & $218(7.5)$ & 147 (11.5) & $501(4.1)$ & $495(5.2)$ \\
\hline Calcium $^{1}$ & $17(1.0)$ & $6(0.6)$ & $5(0.3)$ & $67(1.9)$ & $157(5.4)$ & $72(5.6)$ & $324(2.7)$ & $301(3.2)$ \\
\hline MVMM $^{1}$ & $155(9.1)$ & $50(5.0)$ & $179(10.6)$ & $460(12.9)$ & $300(10.3)$ & $128(10.0)$ & $1272(10.5)$ & 1067 (11.3) \\
\hline Fish oil $^{1}$ & $7(0.4)$ & $7(0.7)$ & $26(1.5)$ & $50(1.4)$ & $42(1.4)$ & $15(1.2)$ & $147(1.2)$ & $133(1.4)$ \\
\hline Fish liver oil ${ }^{2}$ & $4(0.2)$ & $0(0.0)$ & $1(0.1)$ & $10(0.3)$ & $16(0.6)$ & $16(1.3)$ & $47(0.4)$ & $43(0.5)$ \\
\hline \multicolumn{9}{|c|}{ Males } \\
\hline All types & $87(10.2)$ & $33(6.4)$ & $86(10.9)$ & $216(12.9)$ & 179 (13.3) & $94(17.6)$ & 695 (12.2) & $575(13.3)$ \\
\hline Single vitamin $\mathrm{D}$ & $1(0.1)$ & $1(0.2)$ & $6(0.8)$ & $27(1.6)$ & $51(3.8)$ & $36(6.8)$ & $122(2.1)$ & $120(2.8)$ \\
\hline Calcium $^{1}$ & $9(1.1)$ & $3(0.6)$ & $3(0.4)$ & $14(0.8)$ & $19(1.4)$ & $11(2.1)$ & $59(1.0)$ & $47(1.1)$ \\
\hline MVMM $^{1}$ & $75(8.8)$ & $26(5.0)$ & $68(8.7)$ & $161(9.6)$ & $99(7.4)$ & $45(8.4)$ & $474(8.3)$ & $373(8.6)$ \\
\hline Fish oil ${ }^{1}$ & $3(0.4)$ & $4(0.8)$ & $14(1.8)$ & $28(1.7)$ & $19(1.4)$ & $6(1.1)$ & $74(1.3)$ & $67(1.5)$ \\
\hline Fish liver oil ${ }^{2}$ & $2(0.2)$ & $0(0.0)$ & $0(0.0)$ & $5(0.3)$ & $4(0.3)$ & $6(1.1)$ & $17(0.3)$ & $15(0.3)$ \\
\hline \multicolumn{9}{|c|}{ Females } \\
\hline All types & 89 (10.4) & $30(6.1)$ & $138(15.3)$ & $413(21.8)$ & $445(28.4)$ & $229(30.7)$ & $1344(20.8)$ & $1225(24.0)$ \\
\hline Single vitamin $\mathrm{D}$ & $0(0.0)$ & $4(0.8)$ & $20(2.2)$ & 77 (4.1) & $167(10.7)$ & 111 (14.9) & $379(5.9)$ & $375(7.3)$ \\
\hline Calcium $^{1}$ & $8(0.9)$ & $3(0.6)$ & $2(0.2)$ & $53(2.8)$ & $138(8.8)$ & $61(8.2)$ & $265(4.1)$ & $254(5.0)$ \\
\hline MVMM $^{1}$ & $80(9.3)$ & $24(4.9)$ & $111(12.3)$ & $299(15.8)$ & $201(12.8)$ & 83 (11.1) & $798(12.4)$ & $694(13.6)$ \\
\hline Fish oil $^{1}$ & $4(0.5)$ & $3(0.6)$ & $12(1.3)$ & $22(1.2)$ & $23(1.5)$ & $9(1.2)$ & $73(1.1)$ & $66(1.3)$ \\
\hline Fish liver oil $^{2}$ & $2(0.2)$ & $0(0.0)$ & $1(0.1)$ & $5(0.3)$ & $12(0.8)$ & $10(1.3)$ & $30(0.5)$ & $28(0.5)$ \\
\hline
\end{tabular}


Table 2. Characteristics of vitamin D-containing supplement users and non-users among Australian adults $(n=9435)$.

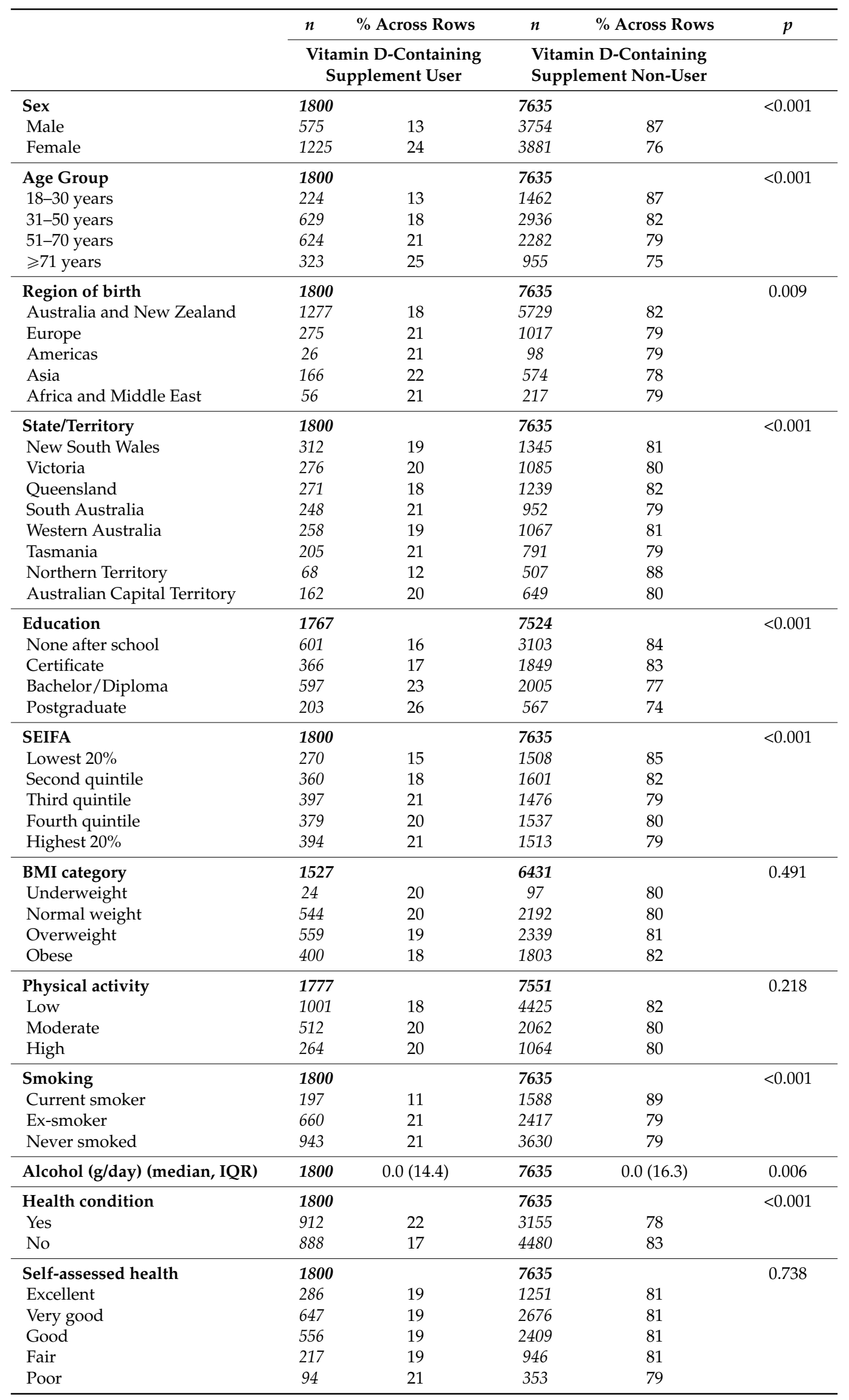


All single vitamin D supplements used by participants provided a daily vitamin D dose of 1000 IU (25 $\mu \mathrm{g}$ ) (Table 3). The median daily dose of vitamin D was $200 \mathrm{IU}(5 \mu \mathrm{g})$ for multivitamin/mineral, calcium and fish oil preparations; and $80 \mathrm{IU}(2 \mu \mathrm{g})$ for fish liver oil. The most commonly used vitamin D-containing supplement type was the multivitamin/mineral (males 8\%; females 12\%) (Table 1). Only $2 \%$ of males and $6 \%$ of females reported using a single vitamin D supplement, but the prevalence increased with age ( $7 \%$ of males and $15 \%$ of females aged $\geqslant 71$ years). In households with one adult and one child/adolescent participant ( $n=2634$ households), children/adolescents were three times more likely to take a vitamin D-containing supplement if the adult was also a vitamin D-containing supplement user (OR 3.2; 95\% CI 2.4, 4.3; $p<0.001$ ).

Table 3. Description of vitamin D dose (IU ( $\mu \mathrm{g}) /$ day) in vitamin D-containing supplements used by survey participants.

\begin{tabular}{ccccc}
\hline Type of Supplement & Minimum & Maximum & Median & IQR \\
\hline Single vitamin D & $1000(25.0)$ & $1000(25.0)$ & $1000(25.0)$ & $0(0.0)$ \\
Multivitamin/mineral & $4(0.1)$ & $1000(25.0)$ & $200(5.0)$ & $224(5.6)$ \\
Calcium preparation & $5.2(1.3)$ & $1000(25.0)$ & $200(5.0)$ & $200(5.0)$ \\
Fish oil preparation & $4(0.1)$ & $1000(25.0)$ & $200(5.0)$ & $264(6.6)$ \\
Fish liver oil & $8(0.2)$ & $252(6.3)$ & $80(2.0)$ & $40(1.0)$ \\
\hline
\end{tabular}

IQR, interquartile range.

Among the users of vitamin D-containing supplements, $93 \%$ of children/adolescents took one vitamin D supplement, while 7\% took two vitamin D-containing supplements on the recording day. Among adult users of vitamin D-containing supplements, $86 \%$ reported taking one, $13 \%$ reported taking two, and $1 \%$ reported taking three or more vitamin D-containing supplements on the recording day. Among the users of vitamin D-containing supplements, 47\%, 24\% and 29\% took a daily dose of $(<400 \mathrm{IU}(<10 \mu \mathrm{g}), 400-999 \mathrm{IU}(10-24.9 \mu \mathrm{g})$ and ( $\geqslant 1000 \mathrm{IU}(\geqslant 25 \mu \mathrm{g})$, respectively. On the recording day, no children/adolescents exceeded the UL for their age group, and $0.1 \%$ of adults (9/9435) exceeded the UL of $4000 \mathrm{IU}(100 \mu \mathrm{g})$.

\subsection{Independent Predictors of Vitamin D-Containing Supplement Use}

Multiple logistic regression analysis in adults showed that females were more than twice as likely as males to use a vitamin D-containing supplement, and the prevalence of use increased significantly with age group (Table 4). Other predictors of vitamin D-containing supplement use included higher educational attainment, higher socio-economic status, greater physical activity and residential location (highest use in those in Tasmania). Those with at least one health condition and poorer self-assessed health were more likely to use a vitamin D-containing supplement. The prevalence of use was higher in all quintiles of SEIFA compared with the lowest quintile, and in ex-smokers and non-smokers compared with current smokers. There were no significant associations between season or BMI category and vitamin D-containing supplement use.

Table 4. Adjusted logistic regression model investigating independent predictors of vitamin D-containing supplement use in Australian adults $(n=7751)$.

\begin{tabular}{lcc}
\hline & Adjusted OR (95\% CI) & $p$ \\
\hline Sex (female $v$ s. male) & $2.05(1.80,2.33)$ & $<0.001$ \\
\hline Age group & & $<0.001$ \\
$18-30$ years & Reference category & 0.002 \\
$31-50$ years & $1.36(1.12,1.64)$ & $<0.001$ \\
$51-70$ years & $2.00(1.60,2.41)$ & $<0.001$ \\
71 years and over & $2.40(1.88,3.05)$ & \\
\hline
\end{tabular}


Table 4. Cont.

\begin{tabular}{|c|c|c|}
\hline & Adjusted OR (95\% CI) & $p$ \\
\hline Sex (female vs. male) & $2.05(1.80,2.33)$ & $<0.001$ \\
\hline Region of birth & & 0.088 \\
\hline Australia and New Zealand & Reference category & \\
\hline Europe & $0.97(0.82,1.15)$ & 0.705 \\
\hline Americas & $1.21(0.75,1.94)$ & 0.435 \\
\hline Asia & $1.26(1.01,1.57)$ & 0.044 \\
\hline Africa and Middle East & $1.38(1.00,1.92)$ & 0.052 \\
\hline State/Territory & & 0.002 \\
\hline New South Wales & Reference category & \\
\hline Victoria & $1.19(0.97,1.46)$ & 0.104 \\
\hline Queensland & $0.99(0.81,1.22)$ & 0.949 \\
\hline South Australia & $1.22(0.98,1.50)$ & 0.074 \\
\hline Western Australia & $1.11(0.90,1.36)$ & 0.344 \\
\hline Tasmania & $1.30(1.04,1.63)$ & 0.023 \\
\hline Northern Territory & $0.64(0.46,0.88)$ & 0.007 \\
\hline Australian Capital Territory & $1.10(0.86,1.40)$ & 0.460 \\
\hline Season & & 0.415 \\
\hline Summer & Reference category & \\
\hline Autumn & $1.12(0.96,1.31)$ & 0.151 \\
\hline Winter & $1.10(0.93,1.30)$ & 0.267 \\
\hline Spring & $1.14(0.96,1.34)$ & 0.150 \\
\hline Education & & $<0.001$ \\
\hline None after school & Reference category & \\
\hline Certificate & $1.27(1.08,1.50)$ & 0.004 \\
\hline Bachelor/Diploma & $1.63(1.40,1.89)$ & $<0.001$ \\
\hline Postgraduate & $1.87(1.44,2.42)$ & $<0.001$ \\
\hline SEIFA & & $<0.001$ \\
\hline Lowest $20 \%$ & Reference category & \\
\hline Second quintile & $1.27(1.04,1.55)$ & 0.017 \\
\hline Third quintile & $1.65(1.35,2.01)$ & $<0.001$ \\
\hline Fourth quintile & $1.31(1.07,1.61)$ & 0.009 \\
\hline Highest $20 \%$ & $1.31(1.06,1.61)$ & 0.012 \\
\hline BMI category & & 0.160 \\
\hline Normal weight & Reference category & \\
\hline Overweight & $0.99(0.86,1.14)$ & 0.876 \\
\hline Obese & $0.85(0.73,1.00)$ & 0.050 \\
\hline Underweight & $1.12(0.70,1.81)$ & 0.638 \\
\hline Physical activity & & 0.006 \\
\hline Low & Reference category & \\
\hline Moderate & $1.09(0.95,1.25)$ & 0.205 \\
\hline High & $1.34(1.12,1.61)$ & 0.002 \\
\hline Smoking & & $<0.001$ \\
\hline Current smoker & Reference category & \\
\hline Ex-smoker & $1.73(1.42,2.10)$ & $<0.001$ \\
\hline Never smoked & $1.58(1.30,1.91)$ & $<0.001$ \\
\hline Alcohol (g/day) & $1.00(1.00,1.00)$ & 0.802 \\
\hline Health condition (yes v no) & $1.24(1.091 .42)$ & 0.001 \\
\hline Self-assessed health & & 0.019 \\
\hline Excellent & Reference category & \\
\hline Very good & $1.22(1.02,1.46)$ & 0.032 \\
\hline Good & $1.31(1.08,1.59)$ & 0.005 \\
\hline Fair & $1.30(1.03,1.66)$ & 0.031 \\
\hline Poor & $1.64(1.19,2.26)$ & 0.003 \\
\hline
\end{tabular}




\subsection{Associations with Serum 25-Hydroxyvitamin D Concentrations}

Participants with measured serum 25(OH)D concentrations were significantly younger $(p<0.001)$ and had a higher level of education $(p<0.001)$ than those without measured 25(OH)D concentrations. There were no significant differences in sex, region of birth and SEIFA in those with and without measured 25(OH)D concentrations.

There was a trend towards higher serum $25(\mathrm{OH}) \mathrm{D}$ concentrations with increasing dose of supplemental vitamin $D$ intake in adults, which was particularly evident in those aged $\geqslant 51$ years (Figure 2). In an unadjusted model, vitamin D intake (per $40 \mathrm{IU}(1 \mu \mathrm{g})$ ) from supplements was associated with higher serum $25(\mathrm{OH}) \mathrm{D}$ concentrations (coefficient $=0.37 ; 95 \% \mathrm{CI} 0.31,0.43 ; p<0.001$ ). The association remained after adjusting for sex, age group, State/Territory, region of birth, season of interview, education, SEIFA, BMI category, physical activity, smoking, alcohol intake, health condition, and self-assessed health: a $40 \mathrm{IU}(1 \mu \mathrm{g})$ increase in vitamin D intake from supplements was associated with an increase of $0.41 \mathrm{nmol} / \mathrm{L}$ in serum $25(\mathrm{OH}) \mathrm{D}$ concentrations $(95 \%$ CI $0.35,0.47 ; p<0.001)$. When stratified by sex, there was a slightly stronger effect in males (Coefficient $=0.49 ; 95 \%$ CI 0.37 , $0.61 ; p<0.001$ ) than in females (Coefficient $=0.38 ; 95 \%$ CI 0.32, $0.45 ; p<0.001$ ). When stratified by season, the association between vitamin $\mathrm{D}$ intake and serum $25(\mathrm{OH}) \mathrm{D}$ concentrations differed marginally depending on season: winter (Coefficient $=0.39 ; 95 \%$ CI $0.27,0.51 ; p<0.001$ ); spring (Coefficient $=0.37 ; 95 \%$ CI 0.25, 0.49; $p<0.001)$; summer (Coefficient $=0.37 ; 95 \%$ CI 0.25, 0.49; $p<0.001)$; autumn (Coefficient $=0.48 ; 95 \%$ CI 0.37, 0.59; $p<0.001$ ).

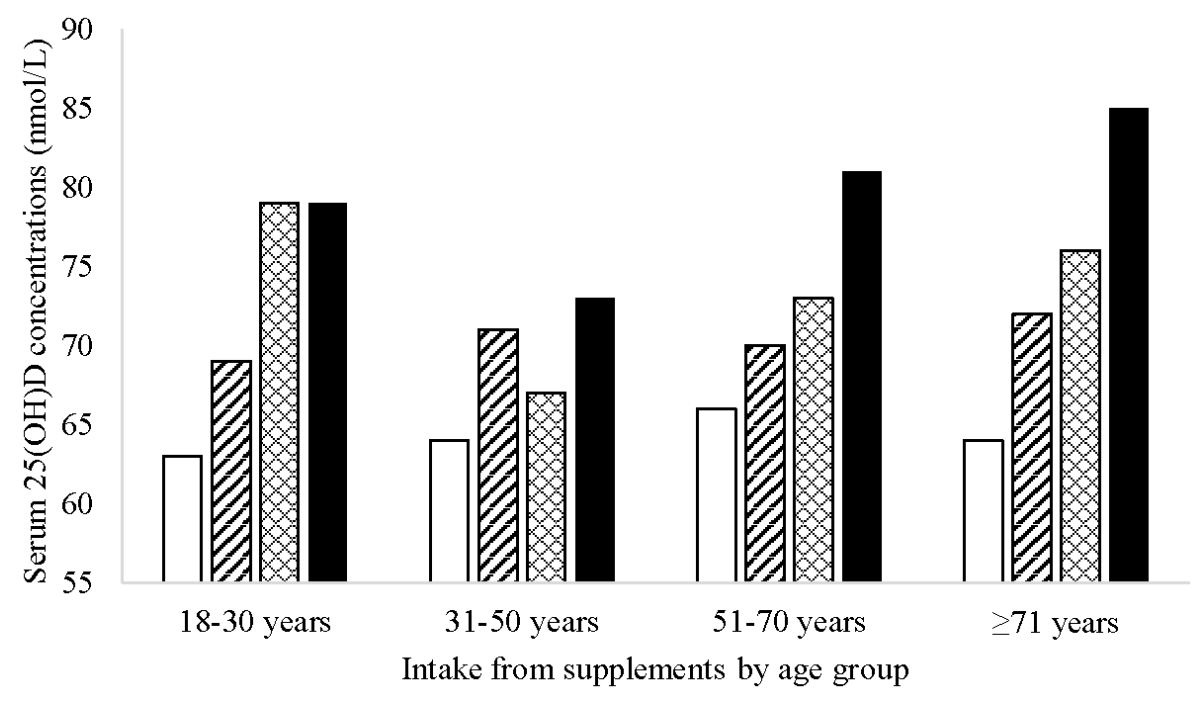

$\square$ No supplement $\boldsymbol{\nabla}<400 \mathrm{IU}(<10 \mu \mathrm{g}) \mathbf{a} 400-999 \mathrm{IU}(10-24.9 \mu \mathrm{g}) \boldsymbol{\square} \geq 1000 \mathrm{IU}(\geq 25 \mu \mathrm{g})$

Figure 2. Mean serum 25-hydroxyvitamin D (25(OH)D) concentrations by vitamin D supplement dose and age group among Australian adults $(n=3736)$.

Vitamin D intake (per $40 \mathrm{IU}(1 \mu \mathrm{g})$ ) was positively associated with vitamin D sufficiency, defined as $\geqslant 50 \mathrm{nmol} / \mathrm{L}$ (OR 1.08 per $40 \mathrm{IU}(1 \mu \mathrm{g})$ increase in supplemental vitamin D; 95\% CI 1.06, 1.10; $p<0.001)$ after adjusting for the potential confounders above. When stratified by sex, there was little difference in the association between vitamin D intake and vitamin D status in males (OR 1.07; 95\% CI 1.04, 1.11; $p<0.001$ ) and females (OR 1.09; 95\% CI 1.07, 1.12; $p<0.001)$. When stratified by season, the association between vitamin $\mathrm{D}$ intake and vitamin $\mathrm{D}$ sufficiency was marginally more pronounced in winter (OR 1.13; 95\% CI 1.08, 1.19; $p<0.001$ ) than spring (OR 1.08; 95\% CI 1.04, 1.12; $p<0.001$ ), summer (OR 1.06; 95\% CI 1.02, 1.11; $p=0.003$ ) and autumn (OR 1.09; 95\% CI 1.05, 1.13; $p<0.001)$. 


\section{Discussion}

This study provides new information on the prevalence and predictors of vitamin D-containing supplement use in the Australian population, based on nationally representative data. We found that $10 \%$ of children, $6 \%$ of adolescents and $19 \%$ of adults reported using a vitamin D-containing supplement. In accordance with other studies $[6,9,18]$, the prevalence of vitamin D-containing supplement use in adults was higher in females than males, with the highest prevalence of use being in older females. The prevalence of vitamin D-containing supplement use reported in the United States (US) and Canada is substantially higher than that reported in our study. For example, in the 2005-2006 National Health and Nutrition Examination Survey (NHANES), vitamin D supplement use in the US ranged from $16 \%$ to $43 \%$ in children/adolescents, and from $21 \%$ to $49 \%$ in adults, depending on sex and age group [13]. In the 2007-2009 Canadian Health Measures Survey (CHMS), $31 \%$ of $6-79$ year olds reported using vitamin D-containing supplements, with the majority of users being female [9]. Both the NHANES and CHMS employed questionnaires that captured vitamin D-containing supplement use in the previous month, as opposed to the single 24-h dietary recall used in our study. Nevertheless, there may genuinely be a higher prevalence of vitamin D-containing supplement use in the US and Canada compared with Australia, since the importance of dietary vitamin $\mathrm{D}$ is emphasised in these countries, with vitamin $\mathrm{D}$ food fortification also widely practiced. In addition, Canada and parts of the US lie at high latitudes where cutaneous synthesis of vitamin $\mathrm{D}$ is limited during winter months, necessitating diet and supplements as an alternative source of vitamin D.

In Ireland, vitamin D-containing supplements were used by $16 \%$ of the adult population based on data derived from a 4-day food record from the 2009 National Adult Nutrition Survey [8]. This is similar to the prevalence observed in our study of Australians. However, in a 7-day food record from the 2004 Irish Children's and 2006 Irish Teens' Food Consumption Surveys, vitamin D-containing supplement use was $21 \%$ in 5-8 year olds, $16 \%$ in $9-12$ year olds and $15 \%$ in $13-17$ year olds [4], which is substantially higher than in our study. This higher prevalence of supplement use amongst children and adolescents may again reflect an awareness of the importance of vitamin D supplementation in a country of high latitude and inclement weather conditions.

Overall, relatively few participants in our study used a single vitamin D supplement-the most commonly used vitamin D-containing supplement type was the multivitamin/mineral. Users of multivitamin/minerals, fish oil and fish liver oil are likely to be taking these supplements for nutritional qualities other than vitamin D. Low dose vitamin D-containing supplements, such as those found in most multivitamin/mineral preparations, are not sufficient to alleviate vitamin D deficiency and may not provide health benefits such as prevention of falls in the elderly. In our study, the highest prevalence of vitamin D-containing supplement use and single vitamin D supplement use was in females aged $\geqslant 51$ years, who are the group with the greatest risk of osteoporosis with increasing age. The greater use of single vitamin D supplements in this population group may reflect a response to recommendations from health professionals to ensure adequate vitamin D status, particularly for reducing risk of falls and fractures. Although nine adults exceeded the UL as defined by the Institute of Medicine as $4000 \mathrm{IU}(100 \mu \mathrm{g})$, some studies have shown no adverse effects with higher daily doses e.g., $6400 \mathrm{IU}(160 \mu \mathrm{g})$ [19] and 10,000 IU (250 $\mu \mathrm{g})$ [17].

Higher level of education and higher SEIFA were associated with vitamin D-containing supplement use in the Australian population. Such a pattern is consistent with that reported in other studies $[9,18]$. This pattern is also apparent in overall dietary supplement use in some countries, including Canada [20] and the US [21,22]. Focus groups in Canada have shown that cost, accessibility, knowledge about what to purchase and lack of information are the most common barriers to supplement use [23]. In Australia, vitamin D supplements are available over-the-counter, and there are no subsidies available for low-income earners who are recommended vitamin D supplements by their general practitioner. 
As with other studies, we found a trend towards higher prevalence of vitamin D-containing supplement use in those who were most physically active. Higher overall dietary supplement use in those who are physically active has been reported in other studies $[22,24]$ and may be due to the perceived benefits of dietary supplements on performance [25] or more health-conscious behaviours in general [26]. Although we found no association between BMI category and vitamin D-containing supplement use, there is evidence of lower serum 25(OH)D concentrations with increased adiposity [27-29].

We found that adults born in Asia were more likely to use vitamin D-containing supplements compared with those born in Australia and New Zealand. Despite the fact that vitamin D deficiency is higher during winter months in most regions of Australia [2], we found no seasonal differences in the prevalence of vitamin D-containing supplement use. The use of vitamin D-containing supplements was least likely among adults living in the Northern Territory and most likely among adults living in Tasmania. This is in line with the relatively low risk of vitamin D deficiency in those residing closer compared to further from the equator (e.g., Northern Territory compared to Tasmania) [2].

As expected, vitamin D intake from supplements was independently associated with higher serum $25(\mathrm{OH}) \mathrm{D}$ concentrations and with greater likelihood of vitamin D sufficiency ( $\geqslant 50 \mathrm{nmol} / \mathrm{L})$, a finding supported by population-based studies in Canada [7,9]. Our results show that a $1000 \mathrm{IU}$ $(25 \mu \mathrm{g})$ dose of supplemental vitamin D (the standard daily dose in a single vitamin D supplement) equates to an average increase in serum $25(\mathrm{OH}) \mathrm{D}$ concentrations of $10 \mathrm{nmol} / \mathrm{L}$, and that those who use a single vitamin D-containing supplement are significantly more likely to be vitamin D sufficient than those who do not take a supplement. It should be noted, however, that although the Institute of Medicine suggest that practically all persons are sufficient at serum $25(\mathrm{OH}) \mathrm{D}$ concentrations of $\geqslant 50 \mathrm{nmol} / \mathrm{L}$, the value of $>75 \mathrm{nmol} / \mathrm{L}$ has been proposed by others [30]. Furthermore, there is some evidence to suggest that concentrations of $75-100 \mathrm{nmol} / \mathrm{L}$ are required for optimal health, particularly in relation to non-skeletal health outcomes [31].

A strength of our study was the availability of comprehensive dietary and demographic data from a nationally representative survey. Supplement intake data were collected directly from participants using face-to-face interview and participants were encouraged to have in front of them any supplements taken. Vitamin D composition data for each product were transcribed from the Australian Register of Therapeutic Goods or from manufacturers' information, ensuring that composition data were accurate at brand and product level. It should be noted, however, that this study was based on supplement intake data from a single 24-h dietary recall, and use on any particular day may not reflect consistent and long-term use of supplements. In addition, we found that adult participants who provided a blood sample for measurement of serum $25(\mathrm{OH}) \mathrm{D}$ concentrations were younger and had a higher level of education than adult participants who did not provide a blood sample. For this reason, associations between supplement dose and serum 25(OH)D concentrations/vitamin D status may not be generalisable to the total population.

\section{Conclusions}

To our knowledge, this is the first detailed investigation of vitamin D-containing supplement use in a nationally-representative sample of the Australian population. Consistent with international populations, the predictors of vitamin D-containing supplement use include higher socio-economic status, higher educational attainment and higher levels of physical activity. We found that the use of vitamin D-containing supplements was independently associated with higher serum $25(\mathrm{OH}) \mathrm{D}$ concentrations and the likelihood of vitamin D sufficiency. However, the prevalence of vitamin D-containing supplement use was generally low in the Australian population, particularly for single vitamin D supplements, with most supplement users obtaining only low levels of vitamin D from other supplement types. Promoting safe levels of sun exposure for optimal health, and introducing food-based strategies to increase vitamin D in the food supply, are likely to include a larger section of the population and may be more effective in reducing the prevalence of vitamin D deficiency in Australia. 
Acknowledgments: The Australian Health Survey was conducted by the Australian Bureau of Statistics (ABS) with funding provided through the ABS health survey program, the Department of Health and the National Heart Foundation of Australia. We thank the Australian Health Survey participants for their time and contribution to the survey. We gratefully acknowledge Paul Atyeo, Beau Stephen and Barry Tynan (ABS) for their advice and assistance in using data from the Australian Health Survey.

Author Contributions: L.J.B. conceived and designed the experiments; L.J.B. and P.J. analyzed the data; L.J.B., C.A.N., R.M.D. and R.M.L. wrote the paper.

Conflicts of Interest: The authors declare no conflict of interest.

\section{Abbreviations}

The following abbreviations are used in this manuscript:

$\begin{array}{ll}\text { 25(OH)D } & \text { 25-hydroxyvitamin D } \\ \text { NNPAS } & \text { National Nutrition and Physical Activity Survey } \\ \text { SEIFA } & \text { Socio-Economic Indexes for Areas } \\ \text { UL } & \text { Tolerable Upper Intake Level }\end{array}$

\section{References}

1. International Agency for Research on Cancer. Globocan 2012: Estimated Cancer Incidence, Mortality and Prevalence Worldwide in 2012. Available online: http://globocan.iarc.fr/Default.aspx (accessed on 2 June 2016).

2. Australian Bureau of Statistics. Australian Health Survey: Biomedical Results for Nutrients; Australian Bureau of Statistics: Canberra, Australia, 2014.

3. Munns, C.F.; Simm, P.J.; Rodda, C.P.; Garnett, S.P.; Zacharin, M.R.; Ward, L.M.; Geddes, J.; Cherian, S.; Zurynski, Y.; Cowell, C.T. Incidence of vitamin D deficiency rickets among Australian children: An Australian paediatric surveillance unit study. Med. J. Aust. 2012, 196, 466-468. [PubMed]

4. Black, L.J.; Walton, J.; Flynn, A.; Kiely, M. Adequacy of vitamin D intakes in children and teenagers from the base diet, fortified foods and supplements. Public Health Nutr. 2013, 17, 721-731. [CrossRef] [PubMed]

5. Fulgoni, V.L.; Keast, D.R.; Bailey, R.L.; Dwyer, J. Foods, fortificants, and supplements: Where do Americans get their nutrients? J. Nutr. 2011, 141, 1847-1854. [CrossRef] [PubMed]

6. Bailey, R.L.; Dodd, K.W.; Goldman, J.A.; Cahche, J.J.; Dwyer, J.T.; Moshfegh, A.J.; Sempos, C.T.; Picciano, M.F. Estimation of total usual calcium and vitamin D intakes in the United States. J. Nutr. 2010, 140, 817-822. [CrossRef] [PubMed]

7. Whiting, S.J.; Langlois, K.A.; Vatanparast, H.; Greene-Finestone, L.S. The vitamin D status of Canadians relative to the 2011 dietary reference intakes: An examination in children and adults with and without supplement use. Am. J. Clin. Nutr. 2011, 94, 128-135. [CrossRef] [PubMed]

8. Black, L.J.; Walton, J.; Flynn, A.; Cashman, K.D.; Kiely, M. Small increments in vitamin D intake by Irish adults over a decade show that strategic initiatives to fortify the food supply are needed. J. Nutr. 2015, 145, 969-976. [CrossRef] [PubMed]

9. Greene-Finestone, L.S.; Langlois, K.A.; Whiting, S.J. Characteristics of users of supplements containing vitamin D in canada and associations between dose and 25-hydroxvitamin D. Appl. Physiol. Nutr. Metab. 2013, 38, 707-715. [CrossRef] [PubMed]

10. Skeie, G.; Braaten, T.; Hjartaker, A.; Lentjes, M.; Amiano, P.; Jakszyn, P.; Pala, V.; Palanca, A.; Niekerk, E.M.; Verhagen, H.; et al. Use of dietary supplements in the european prospective investigation into cancer and nutrition calibration study. Eur. J. Clin. Nutr. 2009, 63, S226-S238. [CrossRef] [PubMed]

11. Gahche, J.; Bailey, R.; Burt, V.; Hughes, J.; Yetley, E.; Dwyer, J.; Picciano, M.F.; McDowell, M.; Sempos, C. Dietary supplement use among US adults has increased since NHANES III (1988-1994). NCHS Data Brief 2011, 61, 1-8. [PubMed]

12. Australian Bureau of Statistics. Australian Health Survey: Users' Guide, 2011-2013; Australian Bureau of Statistics: Canberra, Australia, 2013.

13. Australian Government Department of Health, Therapeutic Goods Administration. Australian Register of Therapeutic Goods. Available online: https://www.tga.gov.au/australian-register-therapeutic-goods (accessed on 2 June 2016). 
14. Sempos, C.T.; Vesper, H.W.; Phinney, K.W.; Thienpont, L.M.; Coates, P.M. Vitamin D status as an international issue: National surveys and the problem of standardization. Scand. J. Clin. Lab. Investig. Suppl. 2012, 243, $32-40$.

15. Australian Bureau of Statistics. Census of Population and Housing: Socio-Economic Indexes for Areas (SEIFA), Australia, 2011. Available online: http://www.abs.gov.au/ausstats/abs@.nsf/Lookup/2033.0.55. 001main+features42011 (accessed on 2 June 2016).

16. Scientific Committee for Food. Guidelines of the Scientific Committee on Food for the Development of Tolerable upper Intake Levels for Vitamins and Minerals; European Commission: Brussels, Belgium, 2000.

17. Institute of Medicine. Dietary Reference Intakes for Calcium and Vitamin D; National Academies Press: Washington, DC, USA, 2011.

18. Green, T.J.; Barr, S.I.; Chapman, G.E. The majority of older British Columbians take vitamin D-containing supplements. Can. J. Public Health 2010, 101, 246-250. [PubMed]

19. Hollis, B.W.; Wagner, C.L.; Howard, C.R.; Ebeling, M.; Shary, J.R.; Smith, P.G.; Taylor, S.N.; Morella, K.; Lawrence, R.A.; Hulsey, T.C. Maternal versus infant vitamin D supplementation during lactation: A randomized controlled trial. Pediatrics 2015, 136, 625-634. [CrossRef] [PubMed]

20. Vatanparast, H.; Adolphe, J.L.; Whiting, S.J. Socio-economic status and vitamin/mineral supplement use in Canada. Stat. Can. 2010, 21, 19-25.

21. Bailey, R.L.; Gahche, J.J.; Lentino, C.V.; Dwyer, J.T.; Engel, J.S.; Thomas, P.R.; Betz, J.M.; Sempos, C.T.; Picciano, M.F. Dietary supplement use in the United States, 2003-2006. J. Nutr. 2011, 141, 261-266. [CrossRef] [PubMed]

22. Radimer, K.; Bindewald, B.; Hughes, J.; Ervin, B.; Swanson, C.; Picciano, M.F. Dietary supplement use by US adults: Data from the national health and nutrition examination survey, 1999-2000. Am. J. Epidemiol. 2004, 160, 339-349. [CrossRef] [PubMed]

23. Whiting, S.J.; Vatanparast, H.; Taylor, J.G.; Adolphe, J.L. Barriers to healthful eating and supplement use in lower-income adults. Can. J. Diet. Pract. Res. 2010, 71, 70-76. [CrossRef] [PubMed]

24. Reaves, L.; Steffen, L.M.; Dwyer, J.T.; Webber, L.S.; Lytle, L.A.; Feldman, H.A.; Hoelscher, D.M.; Zive, M.M.; Osganian, S.K. Vitamin supplement intake is related to dietary intake and physical activity: The Child and Adolescent Trial for Cardiovascular Health (CATCH). J. Am. Diet. Assoc. 2006, 106, 2018-2023. [CrossRef] [PubMed]

25. O'Dea, J.A. Consumption of nutritional supplements among adolescents: Usage and perceived benefits. Health Educ. Res. 2003, 18, 98-107. [CrossRef] [PubMed]

26. Tetens, I.; Biltoft-Jensen, A.; Spagner, C.; Christensen, T.; Gille, M.B.; Bugel, S.; Banke Rasmussen, L. Intake of micronutrients among Danish adult users and non-users of dietary supplements. Food Nutr. Res. 2012, 55. [CrossRef] [PubMed]

27. Drincic, A.T.; Armas, L.A.; van Diest, E.E.; Heaney, R.P. Volumetric dilution, rather than sequestration best explains the low vitamin D status of obesity. Obesity 2012, 20, 1444-1448. [CrossRef] [PubMed]

28. Mai, X.M.; Chen, Y.; Camargo, C.A., Jr.; Langhammer, A. Cross-sectional and prospective cohort study of serum 25-hydroxyvitamin D level and obesity in adults: The hunt study. Am. J. Epidemiol. 2012, 175, 1029-1036. [CrossRef] [PubMed]

29. Snijder, M.B.; van Dam, R.M.; Visser, M.; Deeg, D.J.; Dekker, J.M.; Bouter, L.M.; Seidell, J.C.; Lips, P. Adiposity in relation to vitamin D status and parathyroid hormone levels: A population-based study in older men and women. J. Clin. Endocrinol. Metab. 2005, 90, 4119-4123. [CrossRef] [PubMed]

30. Pludowski, P.; Karczmarewicz, E.; Bayer, M.; Carter, G.; Chlebna-Sokół, D.; Czech-Kowalska, J.; Dębski, R.; Decsi, T.; Dobrzańska, A.; Franek, E.; et al. Practical guidelines for the supplementation of vitamin D and the treatment of deficits in Central Europe-Recommended vitamin D intakes in the general population and groups at risk of vitamin D deficiency. Endokrynol. Polska 2013, 64, 319-327. [CrossRef]

31. Spedding, S.; Vanlint, S.; Morris, H.; Scragg, R. Does vitamin D sufficiency equate to a single serum 25-hydroxyvitamin D level or are different levels required for non-skeletal diseases? Nutrients 2013, 5, 5127-5139. [CrossRef] [PubMed] 